\title{
Solvent Decomposition of Polyurethane Foam Obtained from Waste Upholstered Furniture to Recover Polyols
}

\author{
Yanfang Pang ${ }^{1}$, Xiaosheng Liu ${ }^{1}$, Yan $\mathrm{Li}^{1}$, Tongtong Cui ${ }^{1}$, Xin Liu ${ }^{1}$, Qi Li ${ }^{1,}$, Hui Wan ${ }^{2, *}$, An Mao ${ }^{1,3, *}$ \\ ${ }^{1}$ Key Laboratory of State Forestry Administration for Silviculture of the Lower Yellow River, Shandong Agricultural University, Taian, China \\ ${ }^{2}$ College of Material and Engineering, Southwest Forestry University, Kunming, China \\ ${ }^{3}$ Beijing Key Laboratory of Wood Science and Engineering, Beijing Forestry University, Beijing, China
}

Email address:

wonderfulliqi2364@126.com (Qi Li),wanandwan@yahoo.com (Hui Wan), dannymaoan@126.com (An Mao)

${ }^{*}$ Corresponding author

\section{To cite this article:}

Yanfang Pang, Xiaosheng Liu, Yan Li, Tongtong Cui, Xin Liu, Qi Li, Hui Wan, An Mao. Solvent Decomposition of Polyurethane Foam Obtained from Waste Upholstered Furniture to Recover Polyols. American Journal of Polymer Science and Technology.

Vol. 5, No. 3, 2019, pp. 88-96. doi: 10.11648/j.ajpst.20190503.13

Received: July 11, 2019; Accepted: August 4, 2019; Published: August 15, 2019

\begin{abstract}
The objective of this study was to investigate the feasibility of recovering the polyols from the polyurethane (PU) foam obtained from waste upholstered furniture (sofa) by chemical decomposition using the propylene glycol (PPG) as the decomposing solvent and sodium hydroxide $(\mathrm{NaOH})$ as the catalyst, respectively. The effects of temperature $\left(180^{\circ} \mathrm{C}\right.$ and $\left.200^{\circ} \mathrm{C}\right)$, reaction time $(0.5,1,1.5,2,2.5$, and 3 hours), and PPG/PU weight ratio (2:1, 3:1, and 4:1) on the decomposition reaction and the physicochemical properties of the recovered polyols, such as hydroxyl number, viscosity, and amine number were investigated. Higher temperature and PPG/PU weight ratio promoted the PU decomposition reaction and resulted in lower viscosity, higher hydroxyl number, and higher amine number of the recovered polyols. The hydroxyl number and amine number increased as the reaction progressed, while the viscosity decreased. Once the reaction was completed, little change was observed for the hydroxyl number and viscosity of the recovered polyols. The recovered polyol obtained at $200^{\circ} \mathrm{Cand} \mathrm{PPG} / \mathrm{PU}$ weight ratio of 3:1 showed about the same length of time for the reaction, as well as the physical properties to that obtained at $180^{\circ} \mathrm{C}$ and $\mathrm{PPG} / \mathrm{PU}$ weight ratio of 4:1. These recovered polyols had similar hydroxyl number and viscosity to those of commercial polyol used for PU coatings, elastomers, adhesives, and sealants.
\end{abstract}

Keywords: Polyol, Polyurethane Foam, Upholstered Furniture, Decomposition, Recover

\section{Introduction}

The Polyurethane (PU) foam is a high molecular polymer made of isocyanate and polyol, which are mixed by special equipment and produced by high pressure spraying under the action of foaming agents, catalysts, and other additives. Upholstered furniture is made of fabric, leather, vinyl on the covering and foams and springs for underneath. The increasing use of the PU foams in upholstered furniture makes their recycling a great challenge.

Currently, most of the PU foam wastes are directly burned or go to the landfill creating an environment impact. Only a small fraction of the foams could be recycled appropriately. PU foams can be recycled through various processes, including thermal, mechanical and chemical methods [1-3].
PU foams can be chemically decomposed into feedstock chemicals in the presence of solvent under high temperature with or without catalyst [4]. The solvents used include water, alcohols, inorganic or organic acids, amines, and alkalines [5]. The polyols from the decomposed PU foams can be recovered for formulating new PU products. For example, the recover polyols can be used to prepare polyurethane adhesives for bonding wood composite products [6]. In the decomposition reaction, the choice of solvent, solvent/PU weight ratio, temperature, time, as well as the catalyst type and its concentration can be important factors and worth investigation.

The objective of this study was to investigate the feasibility of recovering polyols from the PU foam wastes obtained from the upholstered furniture (sofa) by chemical decomposition 
using the propylene glycol (PPG) as the decomposing solvent and sodium hydroxide $(\mathrm{NaOH})$ as the catalyst. The effects of the PPG/PU weight ratio, reaction temperature, and time on the decomposition reaction and the physicochemical properties of the recovered polyols, such as hydroxyl number, amine number, and viscosity were investigated.

\section{Materials and Methods}

\subsection{Preparation of PU Powders}

PU foams were obtained from DOTON furniture Inc., Taian. The foams were washed by water and then by acetone (obtained from Sinopharm Group Co. Ltd, Beijing). The foams were then air-dried and grounded into powders by a grounding machine (Laboratory Mill, Model WQM, Jingcheng Chemical Machinery Co. Ltd, Jiangyin) to pass through a $1 \mathrm{~mm}$ screen. The PU powders were then dried at $100^{\circ} \mathrm{C}$ for $1 \mathrm{~h}$.

\subsection{Experimental Variables}

PPG and $\mathrm{NaOH}$ which were used as decomposing solvent and catalyst, respectively, were obtained from Sinopharm Group Co. Ltd, Beijing. PPG is a low viscosity, transparent liquid with a water content of about $0.5 \%$ and a hydroxyl number of about $1276 \mathrm{mg} \mathrm{KOH} / \mathrm{g}$. Two reaction temperatures, $180^{\circ} \mathrm{C}$ and $200^{\circ} \mathrm{C}$, six reaction times, $0.5,1$, $1.5,2,2.5$, and 3 hours, and three PPG/PU weight ratios, 2:1, $3: 1$, and $4: 1$ were used in the experiments (Table 1).

Table 1. Reaction parameters for the decomposition of $P U$.

\begin{tabular}{ll}
\hline PPG/PU weight ratio & $2: 1,3: 1,4: 1$ \\
Reaction temperature $\left({ }^{\circ} \mathrm{C}\right)$ & 180,200 \\
Reaction time $(\mathrm{h})$ & $0.5,1,1.5,2,2.5,3$ \\
Catalyst concentration (based on the & $0.5 \%$ \\
weight of PU powders) & 700 \\
Stirring speed (rpm) & 40 \\
Charging rate (g/min) & 1 \\
System pressure (atm) & 1 \\
FP powder size (mm in diameter) & 0 \\
FP powder moisture content $(\%)$ & \\
\hline
\end{tabular}

\subsection{Decomposition of PU Foams and Recovery of Polyols}

The decomposition reaction was carried out under the atmosphere pressure in a three-neck round-bottom flask equipped with a stirrer and a refluxing condenser (Figure 1). A two-blade PETE impeller was used to stir the reaction mixture in the flask. The PPG was placed in the flask and heated by a heating mantle to $180-200^{\circ} \mathrm{C}$. Then the PU powders and catalyst were charged into the flask. After the charging was completed, the reaction mixture was maintained at $180-200^{\circ} \mathrm{C}$ for $0.5,1,1.5,2,2.5$, and 3 hours. The mixtures obtained from the different reaction conditions were then subjected to the vacuum distillation to remove the excess PPG. The obtained liquids were filtrated through a No. 4 filter paper to obtain the recovered polyols.

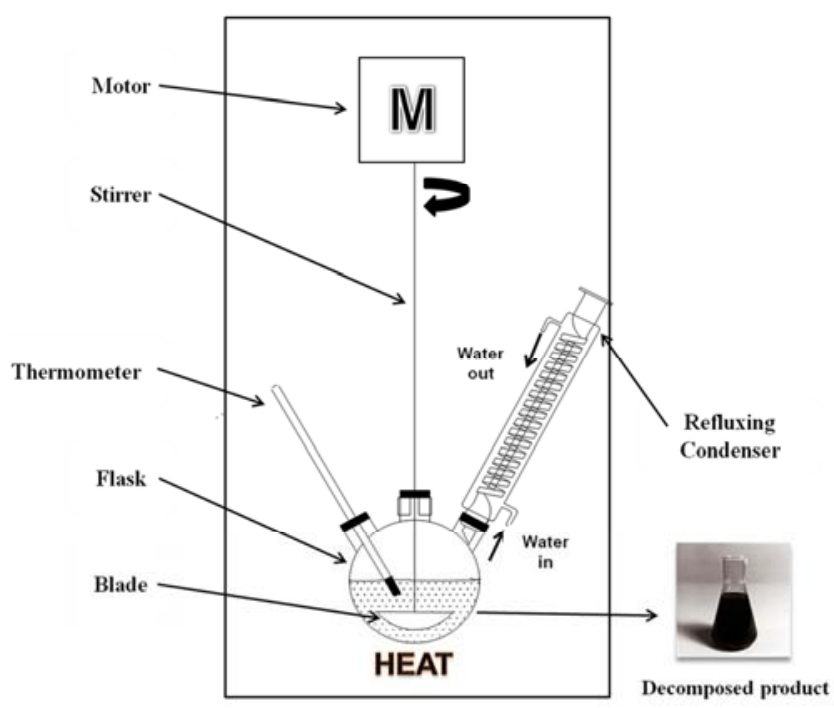

Figure 1. Schematic diagram of decomposition reaction device.

\subsection{Material Characterization}

\subsubsection{Hydroxyl Number}

The hydroxyl number was measured in accordance with the American Oil Chemists' Society (AOCS) Official Method Cd 13-60. Pyridine, acetic anhydride, pyridine-acetic anhydride, normal butyl alcohol, ethanolic potassium hydroxide, phenolphthalein indicator solution were obtained from Sinopharm Group Co. Ltd, Beijing. Three replicates were used and averaged for each measurement. The hydroxyl number was calculated as follows:

Hydroxyl number $=\{[\mathrm{B}+(\mathrm{W} \times \mathrm{A} / \mathrm{C})-\mathrm{S}] \times \mathrm{N} \times 56.1\} / \mathrm{W}(1)$

Where:

A- $\mathrm{KOH}$ solution required for the acidity titration, $\mathrm{mL}$

$\mathrm{B}-\mathrm{KOH}$ solution required for the reagent blank, $\mathrm{mL}$

C- Weight of sample used for the acidity titration, $g$

$\mathrm{S}-\mathrm{KOH}$ solution required for titration of the acetylated sample, $\mathrm{mL}$

W- Weight of sample used for acetylation, $g$

\subsubsection{Viscosity}

The viscosity was measured by a Rotational Brookfield Viscometer (Brookfield Engineering Laboratories. Inc., MA). The measurements were performed at $25^{\circ} \mathrm{C}$.

\subsubsection{Amine Number}

The amine number was measured in accordance with the procedures described in American Society for Testing and Materials (ASTM) D 2073-92 (reapproved 1998). Acetic acid, acetic anhydride, chloroform, hydrochloric acid, isopropanol, perchloric acid, and salicylaldehyde were obtained from Sinopharm Group Co. Ltd, Beijing. Three replicates were used and averaged for each measurement. The total amine number was calculated as follows:

$$
\text { Total amine number }=(\mathrm{V} \times \mathrm{N} \times 56.1) / \mathrm{S}
$$

Where:

V- Hydrochloric acid required for titration of the sample, 
$\mathrm{mL}$

N- Normality of the hydrochloric acid solution

S- Sample weight used, $g$

\subsubsection{Yield}

The yield of the recovered polyols determined by the weight measurement, and was calculated as follows:

$$
\text { Yield } \%=(\mathrm{P} / \mathrm{F}) \times 100 \%
$$

Where:

P- Weight of the recovered polyols, $g$

F- Weight of the PU powders, $g$

\subsubsection{PH Value}

$\mathrm{PH}$ value was determined by an Accumet basic $\mathrm{AB} 15 \mathrm{pH}$ meter (Fisher Scientific Co., PA) at $25^{\circ} \mathrm{C}$.

\subsubsection{Fourier Transform Infrared (FT-IR) Spectroscopy}

FT-IR analysis was conducted using a Thermo Fisher Nicolet 6700 spectrophotometer (Thermo Fisher Scientific Inc. MA). The solid samples of the PU foam powders were located on the stage of the spectrophotometer. The liquid samples of the reaction mixtures were dropped on the stage of the spectrophotometer. FT-IR spectra of a total of 64 scans for each sample from 4000 to $600 \mathrm{~cm}-1$ wavenumber were recorded. The functional groups contained in both the PU powders and the reaction mixtures were examined through the spectra.

\section{Results and Discussion}

\subsection{Characterization of the PU Foams}

The FU foams collected at the furniture factory had a dark yellow color. They were washed by water and acetone, and oven dried. The color of the foams turned to light yellow after washing, and a weight loss of additional $5-10 \%$ was obtained. The weight loss could be from water-soluble and acetone-soluble liquids, and volatile compounds.

The chemical compositions of the grounded PU powders were characterized using the FT-IR technique. The spectrum in Figure 2 shows the absorption bands of the PU powders. The absorption bands at about $1720 \mathrm{~cm}^{-1}$ and $1537 \mathrm{~cm}^{-1}$ corresponds to the urethane carbonyl group $(\mathrm{C}=\mathrm{O}$ in $-\mathrm{NH}-$ $\mathrm{CO}-\mathrm{O}-$ ) stretching and $\mathrm{N}-\mathrm{H}$ bending vibration, respectively. The absorption bands at about $1662 \mathrm{~cm}^{-1}$ and $3310 \mathrm{~cm}^{-1}$ corresponds to the urea carbonyl group $(\mathrm{C}=\mathrm{O}$ in $-\mathrm{NH}-\mathrm{CO}-$ $\mathrm{NH}-)$ and the stretching vibration of N-H groups, respectively. These groups were the products from the reaction between isocyanate and water [7-9]. The absorption band of about $1450 \mathrm{~cm}^{-1}$ corresponding to aromatic ring indicated the possible aromatic source of the isocyanate as one of the raw materials in the preparation of the PU foams [10]. The intense stretching vibration of the aliphatic $-\mathrm{C}-\mathrm{O}-$ $\mathrm{C}$ - group at about $1100 \mathrm{~cm}^{-1}$ suggested that the main type of the raw polyols used in the preparation of the PU foams could be polyether polyols.

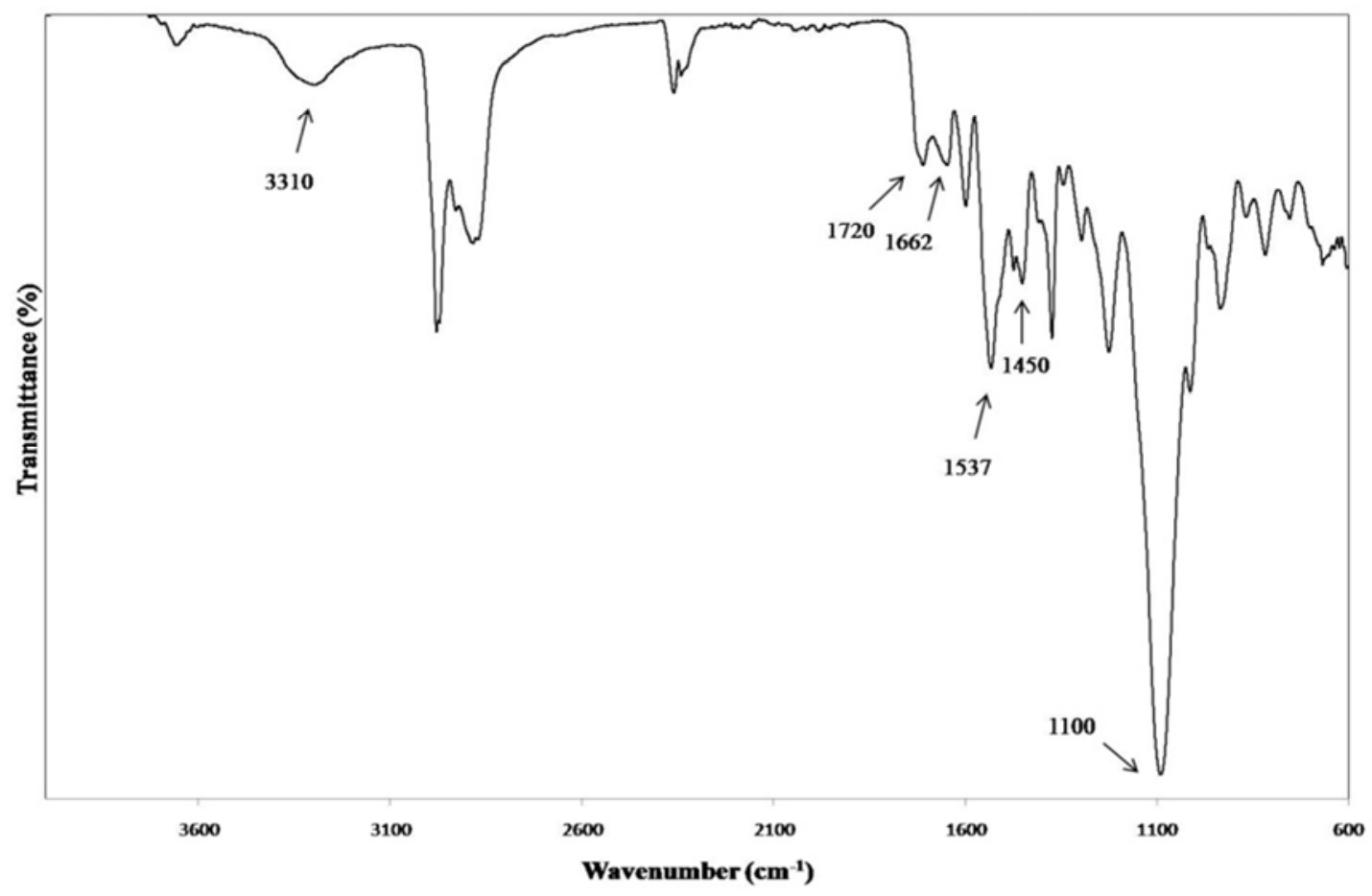

Figure 2. FT-IR spectrum of the PU foam powders obtained from the upholstered furniture (sofa).

\subsection{Decomposition Reaction of the PU Foams by PPG}

\subsubsection{General Observations}

Since the PU powders were in low density and with a hydrophobic nature, it had a tendency to float on the surface of the hot PPG solution. Almost all the PU powders dissolved in the PPG solution within the first 0.5 hour under all reaction conditions. The regular temperatures used in the decomposition reaction of PU materials by glycols were $190-250^{\circ} \mathrm{C}$ [5]. Two reaction temperatures were used in the 
experiments. One was $200^{\circ} \mathrm{C}$, which is among the regular temperature range $\left(190-250^{\circ} \mathrm{C}\right)$. Higher temperature may increase the side reaction rates and produce more by-products [11]. The other temperature was $180^{\circ} \mathrm{C}$. It was observed that the dissolution of the PU powders in the PPG was slower at $180^{\circ} \mathrm{C}$ than that at $200^{\circ} \mathrm{C}$ and the color of the reaction mixtures at $200^{\circ} \mathrm{C}$ was a slightly darker than that at $180^{\circ} \mathrm{C}$.

The decomposition of the PU foams involved intermolecular transesterification of the urethane bonds by the reaction of the hydroxyl end groups with the glycols [12]. As the reaction progressed, the polyurethane bonds were gradually broken down by the PPG and converted into the urethane oligomers (smaller fragments). Phase separation of the reaction mixtures was observed when the PPG/PU weight ratio was $4: 1$ at both reaction temperatures $\left(200^{\circ} \mathrm{C}\right.$ and $\left.180^{\circ} \mathrm{C}\right)$. The phase separation started to occur at the early stage of the reaction and was clearly observed from the sample obtained at a reaction time of 0.5 hours. The reaction mixtures were separated into two liquid phases after cooling down to $25^{\circ} \mathrm{C}$. No phase separation was observed when PPG/PU weight ratio of 2:1 and 3:1 were used. The volume ratio of the upper phase to the bottom phase varied from 1:4 to $1: 6$, with the bottom phase being more viscous. The color of the upper phase was slightly lighter than that of the bottom phase.

\subsubsection{Characterization of the Reaction Mixtures Obtained at Different Reaction Times}

Figure 3 showed the FT-IR spectra of the reaction mixtures obtained at $200^{\circ} \mathrm{C}$ and the PPG/PU weight ratio of 3:1 as a function of reaction time. As shown in Figure 3, a variation of the intensity of the urethane carbonyl groups $(\mathrm{C}=\mathrm{O}$ in $-\mathrm{NH}-\mathrm{CO}-\mathrm{O}-)$ at about $1720 \mathrm{~cm}^{-1}$ was observed indicating that there was an extent of the decomposition reaction occurred. The absorption intensity of the urethane carbonyl groups varied inversely as a function of reaction time. During the first 0.5 hour of reaction, the intensity decreased greatly as a result of the fast reaction. However, a clear band in the spectrum was still observed at the reaction time of 0.5 hour indicating that there was a partial degradation for the sample. As the reaction continues, the intensity of the band was reduced. After 2 hours of reaction, the band was completely disappeared, and the reaction came to stabilized. The FT-IR results indicated that a complete decomposition of the urethane chains required 2 hours at $200^{\circ} \mathrm{C}$ and PPG/PU ratio of 3:1. The intensity of the absorption band of $-\mathrm{OH}$ groups at $3360 \mathrm{~cm}^{-1}$ also increased as a function of reaction time. However, this was not clearly observed in the spectra due to the presence of the excess of the PPG.

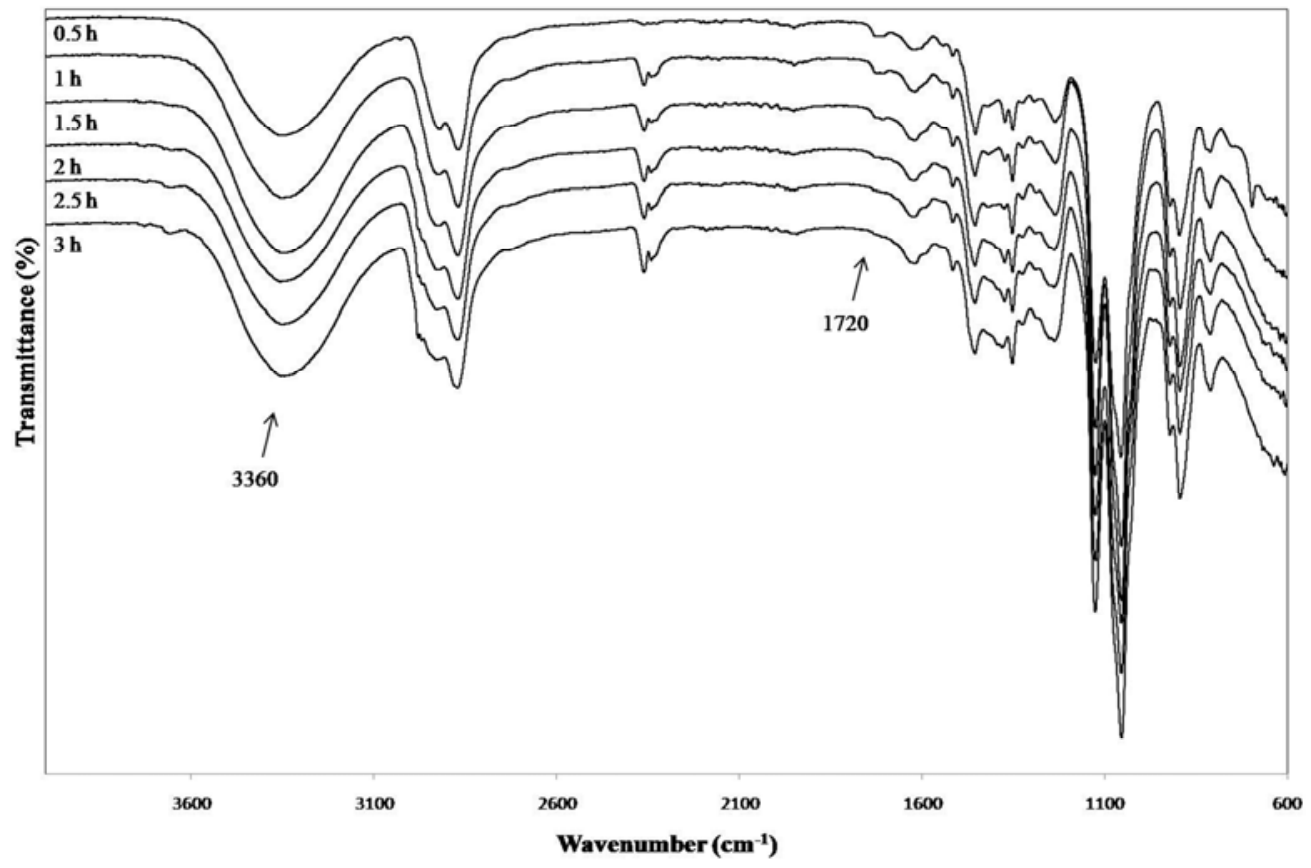

Figure 3. Variation in FT-IR spectrum of the reaction mixtures with time at $210^{\circ} \mathrm{C}$ and PPG/PU ratio of $3: 1$.

\subsubsection{Characterization of the Two Phases from the Reaction Mixture}

Figure 4 shows the FT-IR spectrum of the upper phase from the reaction mixture obtained at $200^{\circ} \mathrm{C}$, reaction time of 3 hours, and PPG/PU weight ratio of $4: 1$. The spectra of two commercially used polyether polyols: BASF Pluracol GP730 and P1010 (BASF Corp., NJ) are used as a comparison. Similar spectra were obtained between the commercially used polyols and that of the upper phase of the reaction mixture indicating that they had the similar chemical structures. This may be due to the presence of the same characteristic absorption bands: (1) intense stretching vibrations of the aliphatic ether groups (-C-O-C-) at about $1100 \mathrm{~cm}^{-1}$, (2) stretching vibrations of the $-\mathrm{OH}$ groups at about 3360-3450 $\mathrm{cm}^{-1}$. Other absorption bands are also normal for the polyols: stretching vibration of $\mathrm{C}-\mathrm{H}$ bonds in aliphatic carbons at $2870-2971 \mathrm{~cm}^{-1}$ and bending vibrations of methylene and methyl groups in the polyol chain at 1374 and $1456 \mathrm{~cm}^{-1}$, respectively [13]. The absorption band of the urethane 
carbonyl groups $(\mathrm{C}=\mathrm{O}$ in $-\mathrm{NH}-\mathrm{CO}-\mathrm{O}-)$ could not be observed due to the complete degradation under this reaction condition. Moreover, it was reported that some solvent in the bottom phase might dissolve in the upper phase [13]. In this study, due to the unknown sources of the PU foams, it was difficult to compare the upper phase with the raw polyols used in the preparation of the PU foams. However, the interference of the solvent should be insignificant so that the spectrum was not affected greatly. Other than these typical bands in the typical polyols, the spectrum of the upper phase did show some slight differences. The presence of the new absorption bands at 1516 and $1628 \mathrm{~cm}^{-1}$ in the spectrum of the upper phase indicated the existence of some amine by-products [11]. Regarding to the intensity of the band of the $-\mathrm{OH}$ groups, the FT-IR spectrum of the upper phase was more similar to that of the GP730 which had higher hydroxyl number (222-237 mg $\mathrm{KOH} / \mathrm{g}$ ) than P1010 (102-112 mg KOH/g). Although a little quantity of PPG might be present in the upper phase and increased intensity of the band of the $-\mathrm{OH}$ groups, it was not readily observed in the FT-IR spectrum.

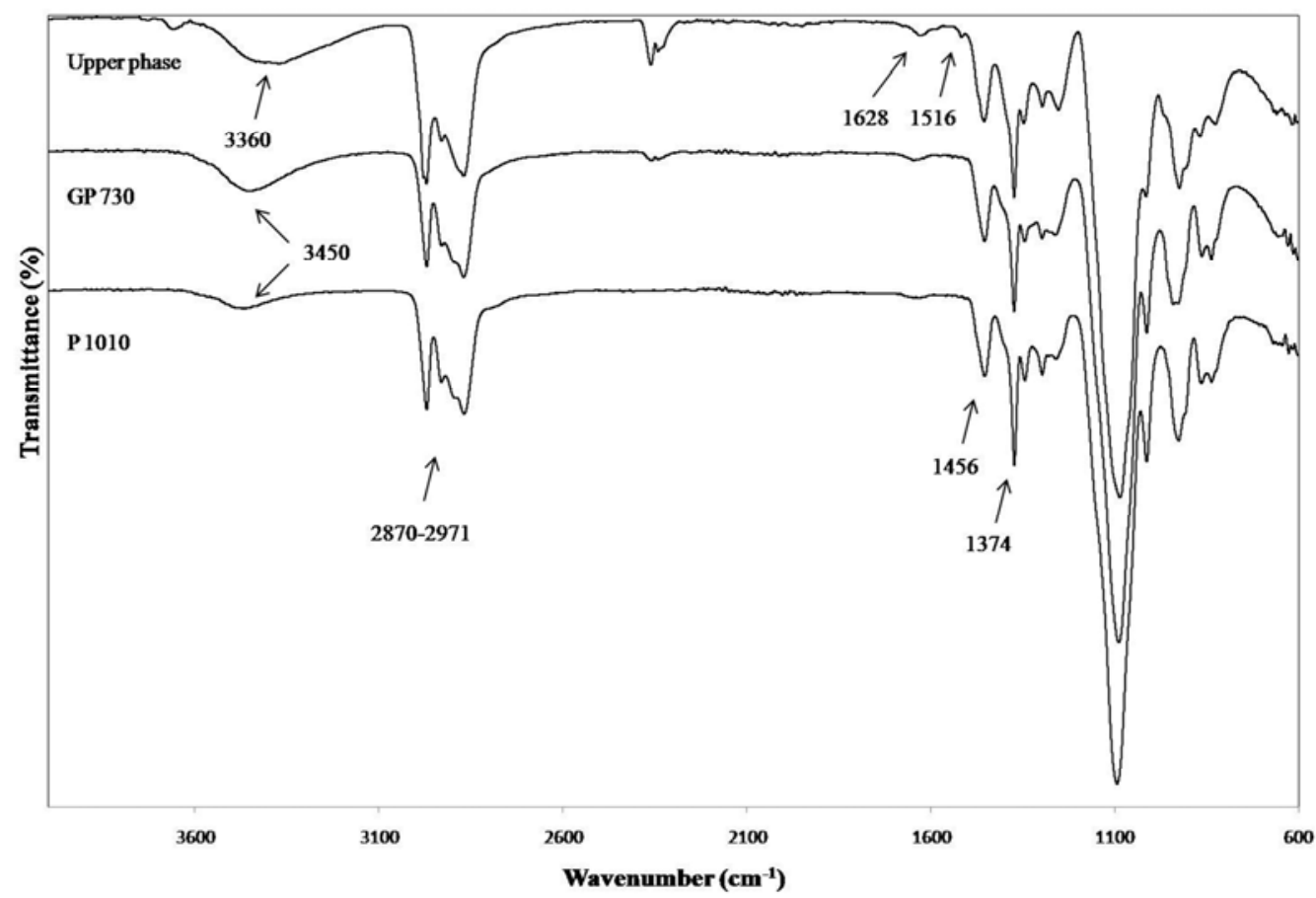

Figure 4. Comparison of FT-IR spectrum between the upper phase of the reaction mixture and commercial polyols.

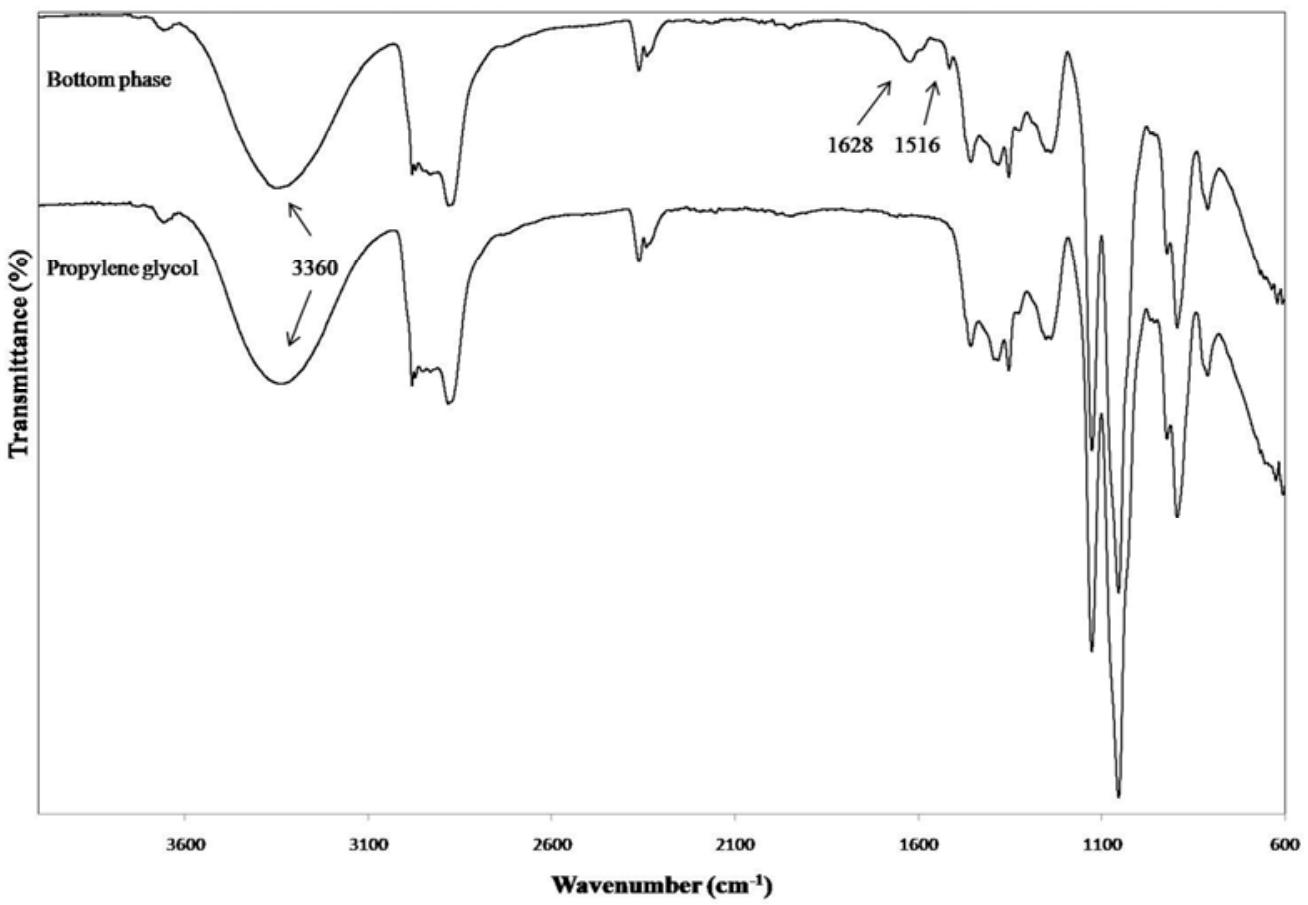

Figure 5. Comparison of FT-IR spectrum between the bottom phase of the reaction mixture and PPG. 
The FT-IR spectrum of the bottom phase was similar to that of PPG (Figure 5). The absorption bands of the $-\mathrm{OH}$ groups with increased intensity were observed at about $3360 \mathrm{~cm}^{-1}$. However, the presence of two new absorption bands at 1516 and $1628 \mathrm{~cm}^{-1}$ with increased intensity in the spectrum of the bottom phase showed some existences of the reaction by-products which are likely to be the amine compounds and their concentration was higher than that in the upper phase. The absorption band of the urethane carbonyl groups $(\mathrm{C}=\mathrm{O}$ in $-\mathrm{NH}-\mathrm{CO}-\mathrm{O}-$ ) at about $1720 \mathrm{~cm}^{-1}$ was absent in the spectrum.

The FT-IR results showed that the upper phase of the reaction mixture was formed mainly by the recovered polyols, and contaminated by a little quantity of the reaction by-products. The bottom phase was formed by the excess PPG and some by-products derived from the starting isocyanates, such as amine compounds. The distribution of amine compounds between the two phases might vary with reaction conditions. The reason for the phase separation could be the polarity difference between the primary hydroxyl groups from PPG and the secondary ones from the polyols [14]. No phase separation was observed when PPG/PU weight ratio of 2:1 and 3:1 were used, indicating that the phase separation was largely influenced by the PPG/PU weight ratio rather than the reaction progress.

\subsection{Effects of the Reaction Conditions on the Properties of the Recovered Polyols}

\subsubsection{Hydroxyl Number}

The hydroxyl number of the decomposed PU foams could be largely influenced by the presence of the excess solvent if the solvent was not separated after reaction [7]. In that case, higher hydroxyl number of the reaction mixtures could be obtained due to the high hydroxyl number of the PPG (1276 $\mathrm{mg} \mathrm{KOH} / \mathrm{g}$ ). In this study, the reaction mixtures obtained from the different reaction conditions were subjected to the vacuum distillation to remove excess PPG. The obtained liquids were filtrated to obtain the recovered polyols with uniform phase. In this way, the influence of the PPG on the hydroxyl number of the recovered polyols was made minimal. Table 2 showed the hydroxyl numbers of the recovered polyols under different reaction conditions. These numbers were in the range of 149-293 $\mathrm{mg} \mathrm{KOH} /$ gfor the samples after 3 hours of reaction. It was noted that within the first $1 \mathrm{~h}$ reaction, the hydroxyl number increased as a function of reaction time due to the substitution of the polyols by the PPG and releasing of polyols. As the reaction progressed, the hydroxyl number became stable for all the samples with the PPG/PU weight ratios of 3:1 and $4: 1$. The results suggested that the adequate reaction time to decompose PU foams under this condition was about 2 hours, which was also confirmed by the variation of the FT-IR spectra with reaction time (Figure 3): the intensity of the absorption band of the urethane carbonyl groups $(\mathrm{C}=\mathrm{O}$ in $\mathrm{NH}-\mathrm{CO}-\mathrm{O}-$ ) decreased with the reaction time and disappeared at 2 hours. It was also observed that lower reaction temperature $\left(180^{\circ} \mathrm{C}\right)$ required more reaction time $(2.5$ hours) to obtain the stable hydroxyl number. Moreover, higher PPG/PU weight ratio resulted in higher hydroxyl number and required less time to obtain the stable hydroxyl number. This is because PPG served both as reactant and solvent in the decomposition reaction of the PU foams. Higher PPG/PU weight ratio enhanced the decomposition reaction and thus shortened the reaction complete time. Therefore, the reactions with a PPG/PU weight ratio of 2:1 may need more than 3 hours to complete the total reaction.

Table 2. Hydroxyl numbers of the recovered polyols at different reaction conditions.

\begin{tabular}{|c|c|c|c|c|c|c|c|}
\hline \multirow{2}{*}{ Reaction temperature $\left({ }^{\circ} \mathrm{C}\right)$} & \multirow{2}{*}{ PPG/PU weight ratio } & \multicolumn{6}{|c|}{ Hydroxyl number (mg KOH/g) } \\
\hline & & $0.5 \mathrm{~h}$ & $1 \mathrm{~h}$ & $1.5 \mathrm{~h}$ & $2 \mathrm{~h}$ & $2.5 \mathrm{~h}$ & $3 \mathbf{h}$ \\
\hline 180 & $2: 1$ & 66 & 77 & 101 & 116 & 131 & 149 \\
\hline 180 & $4: 1$ & 105 & 159 & 209 & 235 & 248 & 252 \\
\hline 200 & $2: 1$ & 73 & 111 & 144 & 164 & 195 & 209 \\
\hline 200 & $3: 1$ & 90 & 155 & 212 & 262 & 250 & 253 \\
\hline 200 & $4: 1$ & 112 & 177 & 255 & 240 & 260 & 293 \\
\hline
\end{tabular}

\subsubsection{Viscosity}

The extent of the decomposition reaction was estimated from the variation of the viscosities with reaction time as shown in Table 3. It was obvious that the viscosity decreased rapidly within the first $1 \mathrm{~h}$ reaction due to the fast reaction rate. Then the change of the viscosity slowed down gradually and reached stable. The viscosity of the recovered polyols decreased from $611-1028 \mathrm{mPa} \cdot \mathrm{s}$ at a reaction time of 0.5 hour to $242-430 \mathrm{mPa} . \mathrm{s}$ at a reaction time of 3 hours. As the reaction progressed, the PU chains were attacked by the PPG, and got broken, and the molecular weight of the polymer thus decreased resulting ina lower viscosity value. A lower PPG/PU weight ratio needed a longer time to obtain a stabled viscosity. The viscosity of the recovered polyols is strongly depended on the reaction temperature. Higher reaction temperature resulted in a lower viscosity. Other than the reaction conditions, the viscosity of the recovered polyols could also be influenced by the residual PPG and other by-products derived from the aromatic segments of the isocyanate [15]. 
Table 3. Viscosities of the recovered polyols at different reaction conditions.

\begin{tabular}{|c|c|c|c|c|c|c|c|}
\hline \multirow{2}{*}{ Reaction temperature $\left({ }^{\circ} \mathrm{C}\right)$} & \multirow{2}{*}{ PPG/PU weight ratio } & \multicolumn{6}{|c|}{ Viscosity $(\mathrm{mPa} \cdot \mathrm{s})$} \\
\hline & & $0.5 \mathrm{~h}$ & $1 \mathrm{~h}$ & $1.5 \mathrm{~h}$ & $2 \mathrm{~h}$ & $2.5 \mathrm{~h}$ & $3 \mathrm{~h}$ \\
\hline 180 & $2: 1$ & 1028 & 797 & 678 & 549 & 455 & 430 \\
\hline 180 & $3: 1$ & 769 & 622 & 421 & 367 & 311 & 320 \\
\hline 180 & $4: 1$ & 666 & 463 & 344 & 287 & 272 & 280 \\
\hline 200 & $2: 1$ & 858 & 668 & 535 & 428 & 400 & 363 \\
\hline 200 & $3: 1$ & 626 & 459 & 348 & 282 & 263 & 275 \\
\hline 200 & $4: 1$ & 611 & 431 & 345 & 249 & 238 & 242 \\
\hline
\end{tabular}

\subsubsection{Amine Number}

Amine number indicates the concentration of the amine compounds. It is an important index for the application the recovered polyols in the PU products [7]. Table 4 shows the effects of the reaction conditions on the amine number of the recovered polyols. Generally, the higher the reaction temperature and PPG/PU weight ratio, and the longer the reaction time were, the higher the amine number would be obtained. The amine compounds could come from the hydrolysis of the urethane groups as a side reaction [16]. The hydrolysis is not desirable in the decomposition reaction, however it is unavoidable. At a temperature around $200^{\circ} \mathrm{C}$, the hydrolysis of the urethane groups by water occurred with the main reaction producing unstable carbamate acids, which was readily decarboxylated and formed aromatic amines [15]. Although most water in the PU foams was removed by oven drying, the PPG which has a water content of up to $0.5 \%$ could bring in water. Higher PPG/PU weight ratio would result in a higher amine number because more water was brought in with PPG. Higher temperature $\left(200^{\circ} \mathrm{C}\right)$ increased the rate of the side reactions and favored the formation of the amine compounds. Another source of the amine compounds could be the decomposition of the urea groups contained in the PU foam backbones. The urea groups were formed by the reaction between water and isocyanate. The water was usually involved in the preparation of the PU foams as surfactant. Although the amount of the urea groups was in a small quantity, they could affect the properties of the PU foams and thus also the properties of the recovered polyols. If the recovered polyols were reused in the preparation of new PU products, the amine compounds might negatively affect the PU foaming reaction. They could accelerate the foaming reaction and form excessive rigid urea groups. Further removing of amine compounds could be done by treating the recovered polyols with monomeric acrylic acid, propylene oxide, glycidyl ether, formaldehyde, and some other chemicals [17, 18]. In this study, because the amine compounds might serve as catalysts for certain PU reactions, the recovered polyols were not further purified. The understanding of the function of the amine compounds for certain PU reactions in the preparation of PU products, such as $\mathrm{PU}$ adhesives will be a future research direction.

Table 4. Amine numbers of the recovered polyols at different reaction conditions.

\begin{tabular}{|c|c|c|c|c|c|c|c|}
\hline \multirow{2}{*}{ Reaction temperature $\left({ }^{\circ} \mathrm{C}\right)$} & \multirow{2}{*}{ PPG/PU weight ratio } & \multicolumn{6}{|c|}{ Amine number (mg KOH/g) } \\
\hline & & $0.5 \mathrm{~h}$ & $1 \mathrm{~h}$ & $1.5 \mathrm{~h}$ & $2 \mathrm{~h}$ & $2.5 \mathrm{~h}$ & $3 \mathbf{h}$ \\
\hline 180 & $2: 1$ & 2.11 & 2.37 & 2.85 & 3.67 & 3.71 & 4.26 \\
\hline 180 & $3: 1$ & 3.12 & 3.43 & 4.25 & 4.75 & 4.87 & 5.48 \\
\hline 180 & $4: 1$ & 3.08 & 3.48 & 4.31 & 4.78 & 5.29 & 5.95 \\
\hline 200 & $2: 1$ & 3.21 & 4.49 & 4.89 & 4.95 & 5.98 & 5.97 \\
\hline 200 & $3: 1$ & 4.31 & 4.64 & 5.07 & 4.79 & 5.96 & 6.08 \\
\hline 200 & $4: 1$ & 4.89 & 4.19 & 6.08 & 5.99 & 6.88 & 6.94 \\
\hline
\end{tabular}

\subsubsection{Yield}

The yields of the recovered polyols are shown in Table 5. As reaction time increased, the yield increased. Same yield was obtained for the samples with PPG/PU weight ratios of
$3: 1$ and $4: 1$. There was no significant difference in yield between the samples obtained at PPG/PU weight ratio of $3: 1$ and 4:1 under the same temperature once the reaction was considered complete.

Table 5. Yields of recovered polyols at different reaction conditions.

\begin{tabular}{|c|c|c|c|c|c|c|c|}
\hline \multirow{2}{*}{ Reaction temperature $\left({ }^{\circ} \mathrm{C}\right)$} & \multirow{2}{*}{ PPG/PU weight ratio } & \multicolumn{6}{|c|}{ Yield (\%) } \\
\hline & & $0.5 \mathrm{~h}$ & $1 \mathrm{~h}$ & $1.5 \mathrm{~h}$ & $2 \mathrm{~h}$ & $2.5 \mathrm{~h}$ & $3 \mathrm{~h}$ \\
\hline 180 & $2: 1$ & 19.5 & 32.0 & 47.8 & 60.2 & 63.9 & 83.4 \\
\hline 180 & $3: 1$ & 33.1 & 59.2 & 71.5 & 85.6 & 118.0 & 125.4 \\
\hline 180 & $4: 1$ & 41.2 & 84.9 & 115.1 & 120.5 & 125.2 & 127.6 \\
\hline 200 & $2: 1$ & 25.5 & 55.1 & 66.8 & 93.0 & 97.9 & 107.0 \\
\hline 200 & $3: 1$ & 39.6 & 77.0 & 85.9 & 100.2 & 123.5 & 128.9 \\
\hline 200 & $4: 1$ & 42.9 & 73.2 & 99.0 & 133.9 & 127.8 & 136.2 \\
\hline
\end{tabular}




\subsubsection{PH Value}

The difference between $\mathrm{pH}$ values of the recovered polyols was negligible and most of the values were obtained in the range of 7.5-8.0.t was noted that the recovered polyol obtained at the PPG/PU weight ratio of $3: 1$ and reaction temperature of $200^{\circ} \mathrm{C}$ showed similar reaction completion time. And the properties of those obtained at the PPG/PU weight ratio of 4:1 and reaction temperature of $180^{\circ} \mathrm{C}$ are similar.

The application of the recovered polyols was introduced in a number of studies, most of which focused on the preparation of the PU foams $[19,20]$. However, either the recovered polyols could only partially $(5-50 \%)$ replace the commercial polyols or the prepared PU foams had deteriorated properties compared with the commercial PU foams. In this study, after compared with the properties of two typical commercial polyols (Table 6), the properties of some obtained recovered polyols were similar to those of the GP730 which have wide uses. been used in the preparation of PU coatings, elastomers, adhesives, and sealants. Therefore, it is proposed that the recovered polyols obtained in this study may be suitable for the preparation of PU coatings, elastomers, adhesives, and sealants.

Table 6. Properties of typical commercial poylols.

\begin{tabular}{lllll}
\hline Commercial polyether polyols & OH number $(\mathbf{m g ~ K O H} / \mathbf{g})$ & Viscosity (mPa.s) & PH value & Uses \\
\hline BASF Pluracol GP730 & $222-237$ & 270 & 6.6 & PU coatings, elastomers, adhesives, and sealants \\
BASF Pluracol P1010 & $102-112$ & 150 & 6.5 & $\begin{array}{l}\text { PU foams, coatings, elastomers, adhesives, and } \\
\text { sealants }\end{array}$ \\
\hline
\end{tabular}

\section{Conclusions}

PU foam wastes from upholstered furniture were decomposed by the PPG as solvent and $\mathrm{NaOH}$ as catalyst under different reaction conditions. The reaction mixtures were vacuum distilled and filtrated to obtain the recovered polyols. The effects of the reaction temperature, time, and PPG/PU weight ratio on the properties of the recovered polyols including hydroxyl number, viscosity, and amine number were investigated. It was found that: (1) higher temperature promoted the PU decomposition reaction and resulted in a lower viscosity, higher hydroxyl number, and higher amine number; (2) higher $\mathrm{PPG} / \mathrm{PU}$ weight ratio shortened the reaction time and resulted in a higher amine number and lower viscosity; (3) the complete reaction time greatly depended on the reaction conditions. The recovered polyol obtained at the PPG/PU weight ratio of $3: 1$ and reaction temperature of $200^{\circ} \mathrm{C}$ showed similar reaction completion time and properties to those obtained at the PPG/PU weight ratio of $4: 1$ and reaction temperature of $180^{\circ} \mathrm{C}$. Some recovered polyols showed similar properties to those of a commercial polyol. The future study will be focused on the utilization of the recovered polyols in the preparation of suitable PU products.

\section{Author Contributions}

The Manuscript was written through contributions of all authors. All authors have given approval to the final version of the manuscript. Yanfang Pang and Xiaosheng Liu contributed equally and should be considered as co-first authors.

\section{Conflicts of Interest}

The authors declare no competing financial interest.

\section{Acknowledgements}

The authors are grateful for the supports of the Fundamental
Research Funds for the Central Universities (Project No. BJFUKF201912), Youth Foundation of Shandong Natural Science Foundation (Project No. ZR2017QC006), Scientific Research Project of Colleges and Universities in Shandong Province (Project No. J17KA147) and National Student Innovation and Entrepreneurship Training Program (Project No. 201810434013).

\section{References}

[1] E. G. Ashton, W. Kindlein, R. Demori, L. H. A. Cândido, and R. Mauler, Recycling polymeric multi-material products through micronization. Journal of Cleaner Production, 116, 268-278, 2016.

[2] D. Simon, A. M. Borreguero, A. Lucas, and J. F. Rodriguez, Recycling of polyurethanes from laboratory to industry, a journey towards the sustainability. Waste Management, 76, 147-171, 2018.

[3] C. C. Tamara, U. Lorena, J. T. Patrycja, S. Rafael, D. Janusz, and E. Arantxa, Thermoplastic polyurethane with glycolysate intermediates from polyurethane waste recycling. Polymer Degradation and Stability, 144, 411-419, 2017.

[4] K. Hamad, M. Kaseem, and F. Deri, Recycling of waste from polymer materials: An overview of the recent works. Polymer Degradation and Stability, 98 (12), 2801-2812, 2013.

[5] G. Behrendt, andB. W. Naber, The recycling of polyurethanes (review). Journal of the University of Chemical Technology and Metallurgy, 44 (1), 3-23, 2009.

[6] Q. Li. M. Li, C. Chen, G. Cao, A. Mao, and H. Wan, Adhesives from polymeric methylene diphenyl diisocyanate resin and recycled polyols for plywood. Forest Products Journal, 67 (3/4), 275-282, 2017.

[7] C. H. Wu, C. Y. Chang, C. M. Cheng, and H. C. Huang, Glycolysis of waste flexible polyurethane foam. Polymer Degradation and Stability, 80 (1), 103-111, 2003.

[8] O. J. Kwon, S. R. Yang, D. H. Kim, and J. S. Park, Characterization of polyurethane foam prepared by using starch as polyol. Journal of Applied Polymer Science, 103 (3), 1544-1553, 2007. 
[9] Z. H. Gao, W. B. Wang, X. Y. Zhao, and M. R Guo, Novel whey protein-based aqueous polymer-isocyanate adhesive for glulam. Journal of Applied Polymer Science, 120 (1), 220-225, 2010 .

[10] S. H. Lee, T. Ohkita, and Y. Teramoto, Polyol recovery from biomass-based polyurethane foam by glycolysis. Journal of Applied Polymer Science, 95 (4), 975-980, 2005.

[11] F. Simioni, M. Modesti, andS. A. Rienzi, Polyol recovery from elastomer polyurethane waste. Cellular Polymers, 6 (6), 27-41, 1987.

[12] H. Ulrich, A. Odinak, B. Tucker, andA. A. R. Sayigh, Recycling of polyurethane and polyisocyanurate foam. Polymer Engineering and Science, 18 (11), 844-848, 1978.

[13] M. M. A. Nikje, M. Mohammad, M. Haghshenas, and A. B. Garmarudi, "Split-phase" glycolysis of flexible PUF wastes and application of recovered phases in rigid and flexible foams production. Polymer-Plastics Technology and Engineering, 46 (3), 265-271, 2007.

[14] C. Molero, A. De Lucas, andJ. F. Rodríguez, Activities of octoate salts as novel catalysts for the transesterification of flexible polyurethane foams with diethylene glycol. Polymer Degradation and Stability, 94 (4), 533-539, 2009.

[15] C. Molero, A. De Lucas, and J. F. Rodríguez, Recovery of polyols from flexible polyurethane foam by "split-phase" glycolysis: Study on the influence of reaction parameters. Polymer Degradation and Stability, 93 (2), 353-361, 2008.

[16] D. Simioni, M. T. Garcia, A. De Lucas, A. M. Borreeguero, andJ. F. Rodríguez, Glycolysis of flexible polyurethane wastes using stannous octoate as the catalyst: study on the influence of reaction parameters. Polymer Degradation and Stability, 98 (1), 144-149, 2013.

[17] M. Gassan, B. Naber, V. Neiss, P. Moeckel, andW. C. Weissflog, Preparation of recyclatepolyols and the use thereof in the preparation of polyurethanes. US Patent. 1994, 5357006.

[18] M. Modesti, F. Simioni, R. Munari, and N. Baldoin, Recycling of flexible polyurethane foams with a low aromatic amine content. Reactive and Functional Polymers, 26 (3), 157-165, 1995.

[19] C. Molero, A. De Lucas, F. Romero, and J. F. Rodriguez, Influence of the use of recycled polyols obtained by glycolysis on the preparation and physical properties of flexible polyurethane. Rodriguez. Journal of Applied Polymer Science, 109 (1), 617-626, 2008.

[20] H. Y. Kim, J. W. Choi, Y. C. Chung, and B. C. Chun, The grafting of recycled polyol from waste polyurethane foam onto new polyurethane and its impact on shape recovery and water vapor permeation. Fibers and Polymers, 18 (5), 842-851, 2017. 